\title{
Safety and Comparative Efficacy of Ultrasound-Guided vs. Fluoro- scopically-Guided Stellate Ganglion Block: A Retrospective Cohort Analysis and Focused Review of Literature
}

\author{
Siddarth Thakur MD, Billy K Huh MD, PhD, Lei Feng, MS, Yun Qing PhD, and Christina Le-Short, MD
}

Background: Stellate ganglion blocks (SGBs) are a valuable diagnostic and therapeutic tool for sympathetically mediated pain syndromes. There are many different techniques used to achieve an effective blockade, but there is limited literature comparing the safety and efficacy of them.

Objectives: We aim to evaluate the incidence of complications and the change in numerical rating scale (NRS) pain scores of 4 groups using varied image-guided techniques: fluoroscopy, ultrasound, fluoroscopy and ultrasound, and no image guidance.

Study Design: This is a retrospective cohort study and focused literature review.

Setting: Auniversity hospital outpatient pain clinic.

Methods: A chart review of all SGBs performed at our institution from September 2010 to August 2014 was performed. Data were collected regarding the patients' age, gender, indication for the procedure, NRS score at one to 3 months and 3 - 6 months, type of image guidance used, and occurrence of a procedure-related complication. A literature review was performed on PubMed in January 2017 using the following search terms: stellate ganglion block and ultrasound, fluoroscopy, safety, and efficacy; the search was made using the English language and human subject filters, yielding 92 results.

Results: No complications occurred in the ultrasound group $(n=19)$, fluoroscopy and ultrasound group $(n=10)$, and no image guidance group $(n=2)$. Two complications occurred in the fluoroscopy group $(n=105 ; 1.9 \%)$. There were no statistically significant differences between the groups in terms of complications $(P$ $=1$ ), change in pain during the one to 3 month time-frame $(P=0.157)$, or change in pain between the $3-6$ month time-frame $(P=0.484)$.

Limitations: The retrospective study design with a small sample size could prove to be a limitation.

Conclusions: No statistically significant difference was detectable between fluoroscopy, ultrasound, fluoroscopy and ultrasound, and no image guidance in terms of safety and efficacy. With this in mind, we cannot make any definitive recommendations about which technique should be used; rather, this decision should be based on clinician preference and clinical scenario.

IRB Approval: The research has been approved by the Institutional Review Board (PA14-0706).

Key words: Stellate ganglion block, techniques, fluoroscopy, ultrasound, sympathetically mediated pain, safety, complications, efficacy
From : University of Texas MD Anderson Cancer Center, Houston, Texas

Author for correspondence: Christina Le-Short, MD Address: University of Texas MD Anderson Cancer Center 1515 Holcombe Blvd., Suite 403

Houston, Texas 77030

E-mail: cle2@mdanderson.org
Stellate ganglion blocks (SGBs) have both diagnostic and therapeutic values for sympathetically mediated pain syndromes of the head, neck, and upper extremity. SGBs are frequently used in patients suffering from pain related to complex regional pain syndrome (CRPS) $(1,2)$, intractable angina pectoralis 
(3), acute herpes zoster (shingles) $(4,5)$, early postherpetic neuralgia $(\mathrm{PHN})(6)$, facial pain $(7,8)$, and cancer pain related to radiation or post-mastectomy (9).

The stellate ganglion is a bundle of sympathetic nerve cell bodies formed by the union of the inferior cervical and first thoracic sympathetic ganglia. It is anatomically located anterior to the neck of the first $\mathrm{rib}$ and the transverse process of the seventh cervical vertebra (C7), medial to the scalene muscle, lateral to the thyroid, esophagus, trachea, recurrent laryngeal nerve and longus colli muscle, superior to the subclavian artery and posterior aspect of the pleura, and posterior to the carotid and vertebral arteries. Given the close proximity of numerous critical structures, a complete understanding of the relevant anatomy is essential prior to performing this procedure (10).

The procedure can be performed using anatomic landmarks without image guidance, although needle position and injectate spread are often unpredictable (11). Therefore, it is prudent to use image guidance with fluoroscopy or ultrasound, both of which improve accuracy and have demonstrated efficacy $(1,9,12)$. Although both techniques are frequently used, there is limited literature comparing fluoroscopic-guided versus ultrasound-guided SGBs regarding safety and efficacy.

To address this gap in the literature, we performed a retrospective medical record review of SGBs and analyzed the complication rate and treatment response. Additionally, we conducted a focused review of the literature evaluating the safety and efficacy of ultrasound-guided and fluoroscopically-guided SGBs. METHODS

We performed the retrospective cohort analysis after obtaining Institutional Review Board approval (PA14-0706). The electronic medical records of all adult patients who underwent a SGB from September 2010 to August 2014 were reviewed. The patients were identified by using the billing code associated with the procedure. All patients who underwent SGBs for a painful condition were included in the analysis. Any patients who underwent the procedure for a non-painful indication (e.g., cardiac arrhythmia) were excluded from the analysis. We collected data regarding the patients' age, gender, medical record number, indication for the procedure, numeric rating scale pain score (NRS, with $0=$ no pain and $10=$ worst pain imaginable) at numerous time points (prior to the procedure, between one and 3 months post-procedure and between 3 and 6 months post-procedure), the type of image guidance used, and the occurrence of a procedure-related complication. The investigators who performed the electronic medical record reviews (S.T. and C.L.) were not involved in the clinical care of any patient who was included in the study.

The patients were grouped by the type of image guidance used; fluoroscopy (group one), ultrasound (group 2), fluoroscopy and ultrasound (group 3), or no image guidance (group 4). Based on the group, we conducted 2 analyses: 1 ) the change in pain score from pre-procedure to post-procedure (average NRS pain score one to 3 months and $3-6$ months) and 2) the occurrence of complications.

The SGBs were all performed in the procedure room in an outpatient clinic of a large academic teaching hospital by medical trainees (i.e., pain fellows or anesthesia residents), using a sterile technique under the supervision of board-certified pain physicians. There are no SGB protocols at our institution and determination of the technique is made by the attending physician.

When performed without image guidance, the patient was placed supine, and the anterior tubercle of the transverse process of the sixth cervical vertebra (C6), also known as Chasaignac tubercle, was palpated at the level of the cricoid cartilage. The vascular structures were retracted laterally, and the needle was introduced and directed inferomedially towards the body of the C6. After contact was made with bone, the needle was withdrawn $1-2 \mathrm{~mm}$, and after a small test dose to ensure that there was no intravascular injection, a $10 \mathrm{~mL}$ mixture of local anesthetic and steroid ( $5 \mathrm{~mL} 0.25 \%$ bupivacaine $+4 \mathrm{~mL} 2 \%$ lidocaine $+1 \mathrm{~mL}$ dexamethasone $10 \mathrm{mg} / \mathrm{mL}$ ) was injected to achieve an effective blockade.

SGB performed under fluoroscopy was done with both the paratracheal and oblique approaches. When using the anterior paratracheal approach, an anteroposterior (AP) view was used to identify the anterior tubercle of the transverse process of C6; a similar needle insertion as the traditional landmark technique was used. After bone was contacted and the needle was withdrawn again $1-2 \mathrm{~mm}, 1 \mathrm{~mL}$ of radiopaque contrast was injected to ensure that there was no intravascular spread and to confirm 
the placement of injectate outside of the longus colli muscle along the sympathetic chain. The volume of injectate ranged between $5-10 \mathrm{~mL}$ of a mixture of local anesthetic and steroid $(4-9 \mathrm{~mL} 0.25 \%$ bupivacaine $+1-2 \mathrm{~mL}$ dexamethasone $10 \mathrm{mg} / \mathrm{mL}$ or $1 \mathrm{~mL}$ triamcinolone $40 \mathrm{mg} / \mathrm{mL}$ ), the amount necessary for an effective block according to physician preference. An alternative oblique approach was introduced by Abdi and colleagues in 2004 (13). After the C6 vertebra had been identified in AP view, the fluoroscope was rotated obliquely on the ipsilateral side to allow visualization of the neural foramina. The needle was then placed at the junction of the $\mathrm{C} 6$ uncinate and transverse process and contrast was injected to ensure adequate placement, then $5 \mathrm{~mL}$ of a mixture of clonidine and local anesthetic (25 mcg clonidine, 4 $\mathrm{mL} 0.25 \%$ bupivacaine) was injected.

SGB performed with ultrasound guidance was performed with the probe and sterile sheath placed near the base of the neck. The transverse process of C6, internal jugular vein, carotid artery, and longus colli muscle were identified, and a regular spinal or echogenic spinal needle was placed using an in-plane approach anterolaterally and deep to the carotid artery. A combination of color Doppler and negative aspiration was used to ensure that there was no vascular needle placement, then $4-5 \mathrm{~mL}$ of a solution containing a mixture of local anesthetic and steroid (3- $4 \mathrm{~mL} 0.25 \%$ bupivacaine, $1 \mathrm{~mL}$ dexamethasone) was injected with direct visualization of hydrodissection of the fascial plane surrounding the ganglion. When both fluoroscopy and ultrasound were utilized, the fluoroscopy was used to confirm appropriate contrast spread, with a similar injectate composition and volume as for ultrasound alone.

A focused literature review was performed on PubMed in January 2017. The following search terms were used: stellate ganglion block and ultrasound, fluoroscopy, safety, and efficacy; the search was made using the English language and human species filters, yielding 92 results. All of the abstracts were reviewed, 78 of the articles were read in entirety, and 47 were found to be relevant for review.

\section{Statistical Analysis}

All statistical analysis was performed using the statistical software SAS Version 9.3 (SAS Institute Inc., Cary, NC). A Fisher's exact test was used to evaluate the association between techniques and complications. A Kruskal-Wallis test was used to evaluate the difference in pain score changes from pre-intervention to one to 3 months and $3-6$ months post-intervention among techniques. The records with missing information were excluded from the analysis.

\section{RESULTS}

A total of 136 procedures performed on 75 patients were included in the analysis. One record was excluded as it was not performed for a pain diagnosis. Each record was treated independently. The patients' ages ranged from 21 to 72 years old with the mean age being 50.2 years old; forty-nine $(65 \%)$ of the patients were women (Table 1). The most common indication for the procedure was CRPS (44.1\%) and the remainder of the indications ranged between 1.5 $-35.3 \%$ (Table 2).

No complications were reported in $98.5 \%$ of the procedures' records; only 2 occurred, both in the fluoroscopy group (Table 3). The complications were both localized hematomas. The association between techniques and complications was not significant $(P-$ value $=1)$

The NRS pain scores ranged from $0-10$; for changes of pain scores we subtracted the pain scores at pre-intervention from the ones between one to 3 months post-intervention and between $3-6$ months post-intervention. The changes of scores ranged from -10 to 7 and -10 to 6 . The mean changes in pain scores between one to 3 months were -0.69 for group one, -2.11 for group 2, 0.2 for group 3, and -3.5 for group 4 . The mean changes in pain scores between $3-6$ months were -0.64 for group one, -1.83 for group $2,-0.42$ for group 3 , and -2 for group 4 . In the pain score analyses 22,55 , and 58 records had missing information on the pain scores at pre-intervention, one to 3 months post-intervention, and $3-6$ months post-intervention, respectively. The changes in pain scores, including mean and standard deviation, are summarized in Table 4. The difference in the pain score changes from pre-intervention to one to 3 months post-intervention among techniques was not significant $(P$-value $=0.1574)$. The difference in the pain score changes from pre-intervention to $3-6$ months post-intervention among techniques was not significant $(P$ - value $=0.4844)$.

\section{DISCUSSION}

Percutaneous SGB is ubiquitously utilized by pain physicians for the diagnosis and treatment of sym- 
Table 1. Demographic data $(n=75)$.

\begin{tabular}{|c|c|}
\hline Age (mean \pm SD) & $50.2(21-72)$ \\
\hline Sex M:F (\%, n) & $35 \%(26): 65 \%(49)$ \\
\hline
\end{tabular}

Table 2. Number of procedures by indication $(n=136)$.

\begin{tabular}{|l|c|}
\hline \multicolumn{1}{|c|}{ Indication } & \% (n) \\
\hline Complex regional pain syndrome & $44.1 \%(60)$ \\
\hline Neuropathic pain & $35.3 \%(48)$ \\
\hline Arm pain & $1.5 \%(2)$ \\
\hline Post-mastectomy pain & $2.9 \%(4)$ \\
\hline Facial pain & $12.5 \%(17)$ \\
\hline Herpes-zoster/post-herpetic neuralgia & $3.7 \%(5)$ \\
\hline
\end{tabular}

Table 3. Number of procedures and complications by technique group $(n=136)$.

\begin{tabular}{|l|c|c|}
\hline \multicolumn{1}{|c|}{ Group } & performed & complications \\
\hline & $\mathbf{\%}(\mathbf{n})$ & $\mathbf{\%}(\mathbf{n})$ \\
\hline Group 1 & $77.2 \%(105)$ & $1.9 \%(2)$ \\
\hline Group 2 & $14 \%(19)$ & $0 \%(0)$ \\
\hline Group 3 & $7.4 \%(10)$ & $0 \%(0)$ \\
\hline Group 4 & $1.5 \%(2)$ & $0 \%(0)$ \\
\hline
\end{tabular}

Group 1 = fluoroscopy, Group 2 = ultrasound, Group 3 = fluoroscopy and ultrasound, Group $4=$ no image guidance.

Table 4. Pain scores (NRS) by group.

\begin{tabular}{|l|c|c|}
\hline \multicolumn{1}{|c|}{ Group } & $\begin{array}{c}\text { 1-3 months post- } \\
\text { procedure }\end{array}$ & $\begin{array}{c}\text { 3-6 months post- } \\
\text { procedure }\end{array}$ \\
\hline & mean $( \pm$ SD) & mean $( \pm$ SD) \\
\hline Group 1 & $-0.69( \pm 2.65)$ & $-0.64( \pm 2.96)$ \\
\hline Group 2 & $-2.11( \pm 2.94)$ & $-1.83( \pm 3.14)$ \\
\hline Group 3 & $0.2( \pm 2.17)$ & $-0.42( \pm 3.77)$ \\
\hline Group 4 & $-3.5( \pm 2.83)$ & $-2(-2)$ \\
\hline
\end{tabular}

Group 1 = fluoroscopy, Group 2 = ultrasound, Group 3 = fluoroscopy and ultrasound, Group $4=$ no image guidance.

pathetically mediated pain. In the current study, we found 2 complications related to inadvertent vascular injury resulting in hematoma formation during fluoroscopic-guided SGB. Both of these hematomas resolved with conservative management and did not necessitate surgical drainage. Further, we did not detect any significant difference between the groups regarding pain relief. Overall, our results suggest that SGBs performed with caution and meticulous technique can be done safely with low complication rates.
Although SGBs are traditionally done via an anterior approach without image guidance (14), advances in imaging and technique have improved the safety and efficacy of the procedure (15). They can be performed with magnetic resonance imaging (MRI) (16-18), computed tomography (CT) $(19,20)$, fluoroscopy, or ultrasound guidance. The most commonly used techniques in pain medicine are fluoroscopy-guided and ultrasound-guided with each having its advantages and disadvantages (21). The following is a brief summary of extant literature regarding both.

Fluoroscopically-guided SGB is routinely performed by pain physicians who find alternative imaging guidance time consuming and expensive and therefore, impractical for everyday use (9). The technique is well established and provides excellent bony delineation facilitating easy identification of the C6 transverse process as the target structure for needle placement (22). It also allows for evaluation of injectate spread and avoidance of intravascular injection with the utilization of radiopaque contrast agents. However, fluoroscopy does not reliably detect soft tissue and neurovascular structures, and given the close proximity to various critical structures, a number of potential complications can arise.

One study used ultrasound to evaluate the needle trajectory for fluoroscopically-guided paratracheal SGB approach and reported the frequency of various anatomic structures being present along the needle path: esophagus (36.7\%), vertebral artery (10 $13.3 \%)$, other artery $(12.3-16.7 \%)$, and the thyroid gland (51.7 -60\%), even after manual lateral retraction (23). We are aware of one unpublished case of direct vascular injection with local anesthetic causing convulsions during fluoroscopically-guided SGB. Other reported complications include hoarseness and dysphagia, presumably due to recurrent laryngeal nerve blockade $(5,22)$, weakness due to brachial plexus blockade $(9,24)$, hematoma due to inadvertent vascular injury $(9,25)$, as well as increased pain and paresthesia $(9,25)$, headache $(25)$, and Horner's syndrome (e.g., ptosis, miosis, and anhidrosis) which is actually an indicator of successful blockade (26). These complications may be decreased by using an oblique approach, as no vasculature structures need to be retracted in order to access the stellate ganglion. A study comparing the 2 fluoroscopic approaches found both to be efficacious but the oblique approach 
to be safer and better tolerated by patients (9). Lastly, as with all fluoroscopically-guided procedures, the risk of radiation exposure exists.

There have been numerous complications related to intravascular injections during non-image guided SGBs, including transient locked-in syndrome $(27,28)$, aphasia and hemiparesis (29), and seizures $(30,31)$. Further, there are reports of respiratory compromise including airway obstruction due to retropharyngeal (32) and cervicomediastinal hematomas (33), bilateral recurrent laryngeal paralysis (34), pneumothorax (26), and even sudden death (35). Although these were observed in cases using the "blind" technique, many advocates for ultrasound cite that similar complications can occur with fluoroscopically-guided procedures due to the inability to reliably identify soft tissue and neurovascular structures.

Accordingly, benefits for ultrasonography include the identification of soft tissue structures, real-time needle advancement, and conformation of injectate spread without exposure to radiation (15). For SGB specifically, the vertebral vessels, thyroid gland, longus colli muscle, nerve roots, and the esophagus can be identified and avoided. Further, ultrasound may improve safety and efficacy given the variability in anatomy $(36,37)$. For example, an estimated $10 \%$ of the population have an aberrant vertebral artery that enters at the level of the $\mathrm{C} 5$ transverse process or higher, rather than the expected C6 level (38). Another potential advantage of ultrasound-guided SGBs is the decreased amount of medication necessary for a successful block due to a more targeted injection near the ganglion (36). This was supported in one prospective blinded control study which found a volume of $2 \mathrm{~mL}$ was sufficient for an efficacious blockade (39). However, some pain physicians have not been well trained in ultrasound-guided interventions and find them technically demanding. They report difficulty interpreting images and the cumbersome technique (using one hand to steer the probe while placing the needle with the other) as barriers to implementing ultrasound into their practice.

Although the evidence does suggest that ultrasoundguided SGB reduces the risk of complications, it does not eliminate the risk altogether (21). Similar to other techniques, small hematomas (40), paresthesias, and hoarseness have been reported (41). More serious complications were seen in one case of arterial in- jection of anesthetic, wherein the authors punctured an arterial structure on an initial attempt, and after 5 minutes, re-inserted the needle and injected local anesthetic intravascularly causing convulsions and respiratory arrest; fortunately, the patient was resuscitated successfully without residual deficits and was sent home the same day (42). In another case, cardiac arrest occurred during ultrasound-guided SGB. It was believed to be due to an injection of local anesthetic into the vertebral or inferior thyroid artery $(43,44)$. Again, the patient was stabilized and discharged from the hospital the next day (44). To avoid similar complications, it is prudent to perform an ultrasound scan prior to needle insertion (45), which allows an appropriate needle trajectory to be visualized and planned to avoid injury to neurovascular structures $(23,46)$. Additionally, vascular injections can be avoided by direct visualization with hydrolocalization (repetitive injections of a small volume of fluid) (47), as well as the use of color flow Doppler; neither of the case reports state that either of these steps were taken.

While there is limited literature comparing the safety of techniques, one prospective randomized trial comparing ultrasound-guided and fluoroscopicallyguided SGBs found less untoward side effects with ultrasound-guided SGBS (1). Another prospective trial comparing ultrasound-guided SGB and "blind" techniques found similar results (12).

\section{Limitations}

The present study has several limitations. First, it is a retrospective analysis; therefore we are restricted by the information that is available in the reviewed medical records. It is possible that a procedure-related complication did occur but was not noticed, nor documented, making the procedures seem safer than they actually are. Second, the number of patients (n $=75)$ and procedures $(n=136)$ are small especially for groups $3(n=10)$ and $4(n=2)$. This factor, in addition to the relatively low incidence of complications $(n=2)$, limits our ability to study them. Therefore, the results may have limited generalizability. Regarding the changes in pain, it is possible that the time-points studied (one to 3 months and 3-6 months) did not appropriately capture the therapeutic effect of the SGB.

\section{CONCLUSION}

SGB is an effective tool for reducing pain related to excess sympathetic nervous system activity. Both 
fluoroscopically-guided and ultrasound-guided SGBs appear to be safe and efficacious, and in this retrospective analysis, no significant differences could be detected between the 2 . Therefore, we are not able to make a definitive recommendation regarding the use of either fluoroscopy or ultrasound to perform the procedure. Instead, we recommend the technique that the clinician is most confident will achieve a successful block without untoward side effects.

Author Contributions: Drs. Thakur, Le-Short, Huh, Qing, and Ms. Feng had full access to all of the data in the study and take responsibility for the integrity of the data and the accuracy of the data analysis. Drs. Huh and Le-Short designed the study protocol. Dr. Thakur managed the literature searches and sum-

\section{REFERENCES}

1. Imani F, Hemati K, Rahimzadeh P, Kazemi MR, Hejazian K. Effectiveness of stellate ganglion block under fluoroscopy or ultrasound guidance in upper extremity CRPS. J Clin Diagn Res 2016; 10:UC09-UC12.

2. Yucel I, Demiraran Y, Ozturan K, Degirmenci E. Complex regional pain syndrome type I: Efficacy of stellate ganglion blockade. J Orthop Traumatol 2009; 10:179-183.

3. Chester M, Hammond C, Leach A. Long-term benefits of stellate ganglion block in severe chronic refractory angina. Pain 2000; 87:103-105.

4. Kim HJ, Ahn HS, Lee JY, Choi SS, Cheong YS, Kwon K, Yoon $\mathrm{SH}$, Leem JG. Effects of applying nerve blocks to prevent postherpetic neuralgia in patients with acute herpes zoster: A systematic review and meta-analysis. Korean J Pain 2017; 30:3-17.

5. Makharita MY, Amr YM, El-Bayoumy Y. Effect of early stellate ganglion blockade for facial pain from acute herpes zoster and incidence of postherpetic neuralgia. Pain Physician 2012; 15:467-474.

6. Rauck RL. Stellate ganglion block. Tech Reg Anesth Pain Manage 2001; 5:88-93.

7. Salvaggio I, Adducci E, Dell'Aquila L, Rinaldi S, Marini M, Zappia L, Mascaro A. Facial pain: A possible therapy with stellate ganglion block. Pain Med 2008; 9:958-962.

8. Shanthanna H. Utility of stellate ganglion block in atypical facial pain: A case report and consideration of its possible mechanisms. Case Report Med 2013; 13:293826.

9. Abbas DN, El Ghafar EMA, Ibrahim WA, Omran AF. Fluoroscopic stellate ganglion block for postmastectomy pain: A comparison of the classic anterior approach and the oblique approach. Clin J Pain 2011; 27:207-213.

10. Narouze S, Vydyanathan A, Patel N. Ultrasound-guided stellate maries of previous related work and wrote the first draft of the manuscript. Drs. Thakur, Le-Short, and Huh provided revision for intellectual content and had final approval of the manuscript.

Disclosure: There was no external funding in the preparation of this manuscript.

Conflict of Interest: There was no external funding in the preparation of this manuscript. Each author certifies that he or she, or a member of his or her immediate family, has no commercial association (i.e., consultancies, stock ownership, equity interest, patent/licensing arrangements, etc.) that might pose a conflict of interest in connection with the submitted manuscript ganglion block successfully prevented esophageal puncture. Pain Physician 2007; 10:747-752.

11. Elias M. Cervical sympathetic and stellate ganglion blocks. Pain Physician 2000; 3:294-304.

12. Yoo SD, Jung SS, Kim H-S, Yun DH, Kim DH, Chon J, Hong DW. Efficacy of ultrasonography guided stellate ganglion blockade in the stroke patients with complex regional pain syndrome. Ann Rehabil Med 2012; 36:633-639.

13. Abdi S, Zhou Y, Patel N, Saini B, Nelson J. A new and easy technique to block the stellate ganglion. Pain Physician 2004; 7:327331.

14. Moore DC, Bridenbaugh LD Jr. The anterior approach to the stellate ganglion: Use without a serious complication in two thousand blocks. JAMA 1956; 160:158-162.

15. Narouze S. Ultrasound-guided stellate ganglion block: Safety and efficacy. Curr Pain Headache Rep 2014; 18:424.

16. Slappendel R, Thijssen H, Crul B, Merx JL. The stellate ganglion in magnetic resonance imaging: A quantification of the anatomic variability. Anesthesiology 1995; 83:424-426.

17. Hogan QH, Erickson SJ, Haddox DJ, Abram SE. The spread of solutions during stellate ganglion block. Reg Anesth 1992; 17:78-83.

18. Hogan $\mathrm{QH}$, Erickson SJ. MR imaging of the stellate ganglion: Normal appearance. AJR Am J Roentgenol 1992; 158:655-659.

19. Erickson SJ, Hogan QH. CT-guided injection of the stellate ganglion: Description of technique and efficacy of sympathetic blockade. Radiology 1993; 188:707-709.

20. Christie JM, Martinez CR. Computerized axial tomography to define the distribution of solution after stellate ganglion nerve block. J Clin Anesth 1995; 7:306-311.

21. Perrine DC, Votta-Velis G, Borgeat A. Ultrasound indications for chronic pain management: An update on the most recent evi- 
dence. Curr Opin Anesthesiol 2016; 29:600-605.

22. Jadon A. Revalidation of a modified and safe approach of stellate ganglion block. Indian J Anaesth 2011; 55:52-56.

23. Siegenthaler A, Mlekusch S, Schliessbach J, Curatolo M, Eichenberger U. Ultrasound imaging to estimate risk of esophageal and vascular puncture after conventional stellate ganglion block. Reg Anesth Pain Med 2012; 37:224-227.

24. Serna-Gutiérrez J. Ultrasound-guided stellate ganglion block. Rev Colomb Anestesio 2015; 43:278-282.

25. van Eijs F, Geurts J, van Kleef M, Faber CG, Perez RS, Kessels AG, Van Zundert J. Predictors of pain relieving response to sympathetic blockade in complex regional pain syndrome type 1. Anesthesiology 2012; 116:113-121.

26. Abdi S, Zhou Y, Doshi R, Patel N. Stellate ganglion block: emphasis on the new oblique fluoroscopic approach. Tech Reg Anesth Pain Manage 2005; 9:73-80.

27. Dukes RR, Alexander LA. Transient locked-in syndrome after vascular injection during stellate ganglion block. Reg Anesth Pain Med 1993; 18:378-380.

28. Chaturvedi A, Dash HH. Locked-in syndrome during stellate ganglion block. Indian J Anaesth 2010; 54:324-326.

29. Scott DL, Ghia JN, Teeple E. Aphasia and hemiparesis following stellate ganglion block. Anesth Analg 1983; 62:1038-1040.

30. Kozody R, Ready LB, Barsa JE, Murphy TM. Dose requirement of local anaesthetic to produce grand mal seizure during stellate ganglion block. Can Anaesth Soc J 1982; 29:489-491.

31. Mahli A, Coskun D, Akcali D. Aetiology of convulsions due to stellate ganglion block: A review and report of two cases. Eur J Anaesthesiol 2002; 19:376-380.

32. Higa K, Hirata K, Hirota K, Nitahara K, Shono S. Retropharyngeal hematoma after stellate ganglion block: Analysis of $27 \mathrm{pa}-$ tients reported in the literature. Anesthesiology 2006; 105:12381245.

33. Takanami I, Abiko T, Koizumi S. Life-threatening airway obstruction due to retropharyngeal and cervicomediastinal hematomas following stellate ganglion block. Thorac Cardiovasc Surg 2009; 57:311-312.

34. Wallace MS, Milholland AV. Contralateral spread of local anesthetic with stellate ganglion block. Reg Anesth 1993; 18:55-59.

35. Kashiwagi M, Ikeda N, Tsuji A, Kudo K. Sudden unexpected death following stellate ganglion block. Leg Med (Tokyo) 1999; 1:262-265
36. Janik JE, Hoeft MA, Ajar AH, Alsofrom GF, Borrello MT, Rathmell JP. Variable osteology of the sixth cervical vertebra in relation to stellate ganglion block. Reg Anesth Pain Med 2008; 33:102108.

37. Huntoon MA. The vertebral artery is unlikely to be the sole source of vascular complications occurring during stellate ganglion block. Pain Pract 2010; 10:25-30.

38. Matula C, Trattnig S, Tschabitscher M, Day JD, Koos WT. The course of the prevertebral segment of the vertebral artery: Anatomy and clinical significance. Surg Neurol 1997; 48:125-131.

39. Lee MH, Kim KY, Song JH, Jung HJ, Lim HK, Lee DI, Cha YD. Minimal volume of local anesthetic required for an ultrasoundguided SGB. Pain Med 2012; 13:1381-1388.

40. Abdel Dayem OT, Saeid MM, Ismail OM, El Badrawy AM, Abdel Ghaffar NA. Ultrasound guided stellate ganglion block in postmastectomy pain syndrome: A comparison of ketamine versus morphine as adjuvant to bupivacaine. J Anesthesiol 2014; 2014:792569.

41. Shankar H, Simhan S. Transient neuronal injury followed by intravascular injection during an ultrasound guided stellate ganglion block. Anesth Pain Med 2013; 2:134-137.

42. Fujiwara $\mathrm{S}$, Komasawa $\mathrm{N}$, Kido $\mathrm{H}$, Minami T. A rare case of accidental arterial local anesthetic injection under ultrasound-guided stellate ganglion block. J Clin Anesth 2016; 29:3-4.

43. Nix CM, Harmon DC. Avoiding intravascular injection during ultrasound-guided stellate ganglion block. Anaesthesia 2011; 66:134-135.

44. Rastogi S, Tripathi S. Cardiac arrest following stellate ganglion block performed under ultrasound guidance. Anaesthesia 2010; 65:1042.

45. Peng PW, Narouze S. Ultrasound-guided interventional procedures in pain medicine: A review of anatomy, sonoanatomy, and procedures: Part I: Nonaxial structures. Reg Anesth Pain Med 2009; 34:458-474.

46. Park JH, Min YS, Chun SM, Seo KS. Effects of stellate ganglion block on breast cancer-related lymphedema: Comparison of various injectates. Pain Physician 2015; 18:93-99.

47. Bloc S, Ecoffey C, Dhonneur G. Controlling needle tip progression during ultrasound-guided regional anesthesia using the hydrolocalization technique. Reg Anesth Pain Med 2008; 33:382383. 